\title{
Caracterización de daño por "b-value" de eventos de emisión acústica en ensayos de rotura de roca andesita
}

Dino Alberto Filipussi ${ }^{1,2}$

\author{
${ }^{1}$ Centro Internacional para Estudios de la Tierra ICES, Comisión Nacional de Energía Atómica CNEA, Centro Atómico \\ Constituyentes, Av. Gral. Paz 1499, Buenos Aires, Buenos Aires, Argentina \\ ${ }^{2}$ Departamento de Física, Escuela de Ciencia y Tecnología, Universidad Nacional de San Martín, Martín de Irigoyen \\ 3100, Buenos Aires, Buenos Aires, Argentina. \\ e-mail: filipuss@cnea.gov.ar
}

\section{RESUMEN}

La emisión acústica es un fenómeno por el cual se generan ondas elásticas en un material al ser sometido a esfuerzos. Estas ondas se propagan hasta la superficie del material donde son recibidas por sensores que las transforman en señales eléctricas para su posterior análisis.

En el presente trabajo se analizaron las señales de emisión acústica para evaluar la cantidad de daño acumulado en un material cuando es sometido a esfuerzos y se lo lleva hasta la rotura.

La ley de Gutenberg-Richter predice un comportamiento potencial, dado por el b-value, entre el número acumulado de eventos de emisión acústica y la amplitud de los mismos. A partir de mediciones de emisión acústica efectuadas en trabajos previos, en probetas de andesita sometidas a compresión uniaxial hasta la rotura, se realizó un análisis del progreso del b-value con el objetivo de caracterizar el daño producido en el material. Se obtuvo un $b$-value de $b=1.105$ con un buen coeficiente de correlación de 0.995 . El valor obtenido del b-value es el esperado que oscila entre 0.5 y 1.5. El análisis de las señales permitió correlacionar el cambio del b-value con los valores máximos relativos de los parámetros de emisión acústica.

Palabras clave: Emisión Acústica, Daño en materiales, b-value, Andesita.

\begin{abstract}
Acoustic emission is a phenomenon that produces elastic waves when materials are subjected to tension. These waves spread towards the material surface where they are received by sensors that convert them into electrical signals for further analysis.

In this work, acoustic emission signals were analysed in order to evaluate the amount of accumulated damage in a material when it is subjected to tensions that make it to fracture.

The Gutenberg-Richter law predicts a potential behaviour, given by b-value, between the cumulative number of acoustic emission events and the amplitude of them. Taking account of acoustic emission signals obtained in previous works, in andesite samples subjected to uniaxial compression up to break, an analysis of the progress of $b$-value was done in order to characterize the material damage occurred. A b-value of $b=1.105$ was obtained with a good correlation coefficient of 0.995 . The value obtained from the b-value is the expected value that ranges between 0.5 and 1.5. The analysis of the signals allowed to correlate the change of the bvalue with the relative maximum values of the acoustic emission parameters.
\end{abstract}

Keywords: Acoustic emission, Damage in materials, b-value, Andesite.

\section{INTRODUCCIÓN}

Los materiales sometidos a esfuerzos generan en su interior ondas elásticas producidas por la misma deformación del material y otras fuentes como las debidas al crecimiento de fisuras, movimiento de dislocaciones, rotura de inclusiones, etc. La técnica de emisión acústica permite obtener información de estos procesos midiendo estas ondas elásticas con sensores piezoeléctricos que transforman la señal mecánica en señal eléctrica. Las ondas elásticas provenientes del interior del material son registradas sobre la superficie del material donde se ubican los sensores piezoeléctricos. Estas señales luego son procesadas para su posterior análisis. La 
señal eléctrica de emisión acústica de muy baja amplitud $(10 \mu \mathrm{V})$ y de alta frecuencia $(1 \mathrm{kHz}$ a $1 \mathrm{MHz})$ se caracteriza por eventos discretos con forma de pulsos oscilantes que alcanzan una amplitud máxima en un tiempo de subida (rise time) de la señal y luego esta se amortigua en el tiempo, ver Figura1.

El equipo de emisión acústica, ver Figura 2, cuenta con una placa electrónica que se encarga de la toma de las amplitudes de la señal en función del tiempo pudiéndose ver online la forma de onda de la señal; más tarde la placa procesa esta información y computa los valores de los parámetros característicos de la señal: energía; valor RMS; amplitud máxima, en adelante simplemente amplitud; rise time; duración del evento; etc., Esta técnica de emisión acústica proporciona un método para caracterizar los daños que sufre el material cuando es llevado a la rotura y da cuenta, entre otros, de los procesos de microfisuración, crecimiento de fisuras y finalmente la coalescencia de fracturas con la rotura final del material.

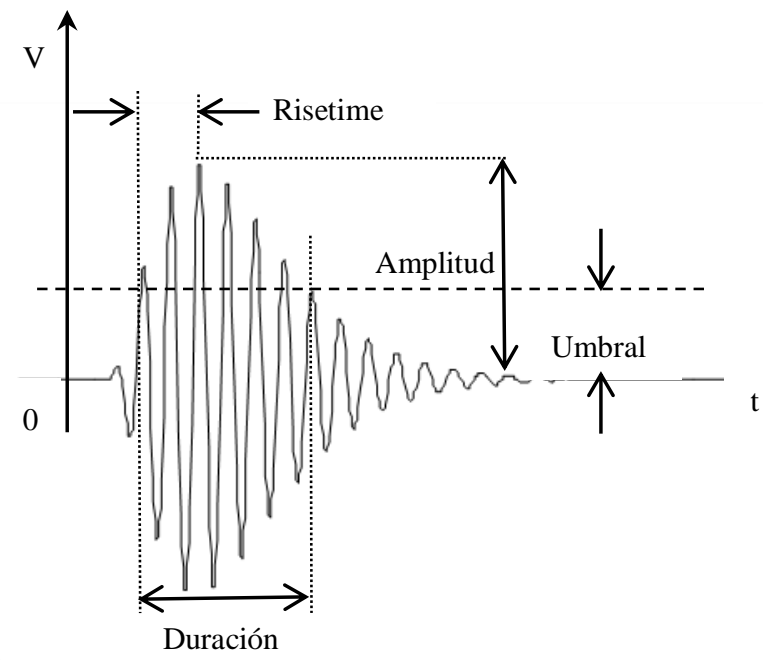

Figura 1: Señal de emisión acústica y algunos parámetros que caracterizan a la misma.

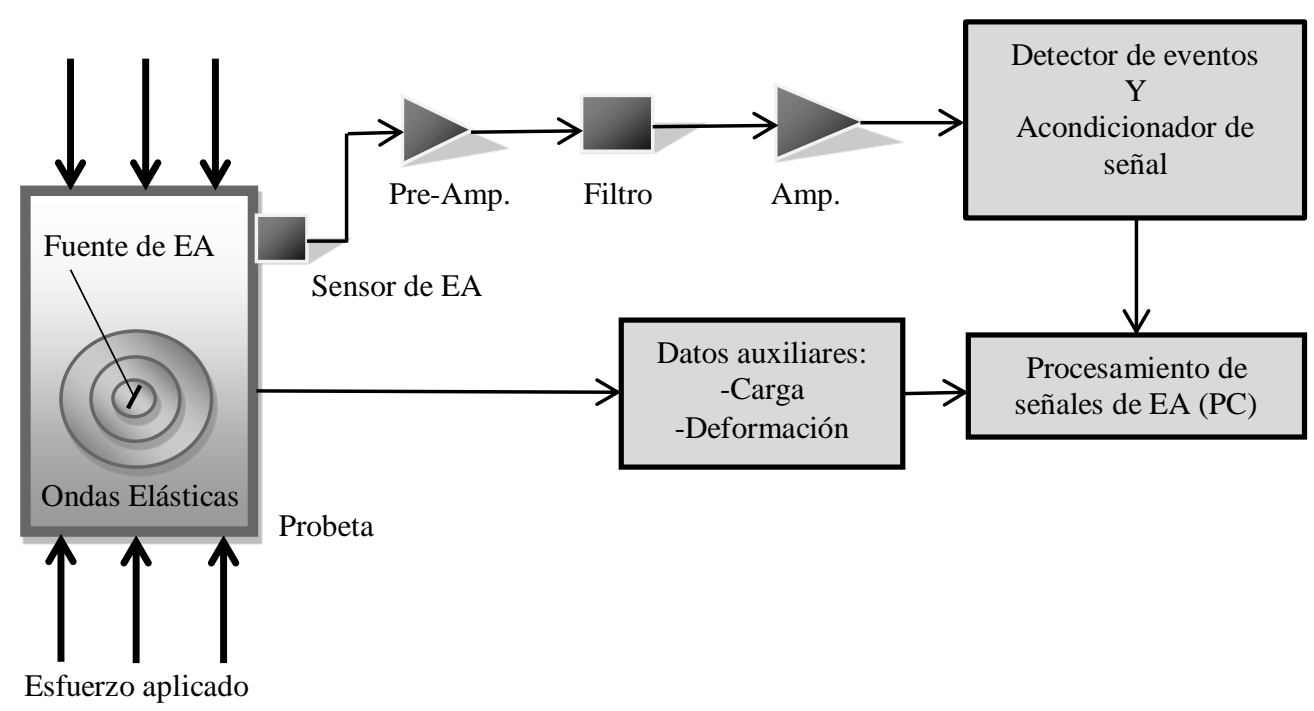

Figura 2: Equipo de Emisión Acústica (EA) y adquisición y procesamiento de datos

En este artículo se analizaron los parámetros que caracterizan a los eventos de emisión acústica y se los comparó con los cambios del b-value. Se quiere caracterizar el daño que sufre el material para un ensayo de compresión uniaxial de una probeta cilíndrica de roca andesita llevada hasta la rotura en una máquina de ensayo industrial realizada en ensayos previos a este trabajo.

Sobre el tema de fractura en un material rocoso sometido a compresión hay numerosos artículos [1-4]. Es interesante observar que la emisión acústica es generada en distintas escalas espaciales y temporales abarcando desde la ruptura de ligaduras atómicas a fallas sísmicas, por ello la información sísmica y la emisión acústica son complementarias, tanto en sus aplicaciones como en su base teórica. A partir de esta idea, para dar cuenta de los procesos de daño en un material rocoso sometido a esfuerzos, hay trabajos [5-9] que estu- 
dian el valor del b-value como resultado de la distribución de frecuencia acumulada de hits de emisión acústica en función de la amplitud de los mismos. Se comprueba que la relación en escala log-log es lineal y es conocida su aplicación en la sísmica como ley de Gutenberg-Richter.

\section{MATERIALES Y MÉTODOS}

En trabajos anteriores [10] de nuestro grupo de investigación se ensayaron cuatro probetas cilíndricas de 75 $\mathrm{mm}$ de diámetro y $150 \mathrm{~mm}$ de largo, realizadas sobre testigos de andesita extraídos in situ de roca del Cerro Blanco, Pcia. San Juan, Argentina. Estas probetas fueron ensayadas a compresión simple uniaxial llevándolas a la rotura. En el presente trabajo se tomaron las mediciones de estos ensayos para procesar y analizar las señales de emisión acústica con el objetivo de determinar los parámetros característicos de los eventos de emisión acústica y calcular los cambios del valor del b-value durante el proceso de fractura. Se utilizó una maquina marca CGTS de 100 toneladas de capacidad de tipo servo hidráulica y lazo cerrado donde se impuso una velocidad de desplazamiento de actuador de $0.12 \mathrm{~mm} / \mathrm{min}$. La Figura 3 muestra el arreglo experimental de una probeta de andesita instrumentada con sensores de emisión acústica. Se lubricaron las caras extremas de las probetas para minimizar la fricción con los cabezales de la máquina y lograr un estado de solicitación lo más uniaxial posible.
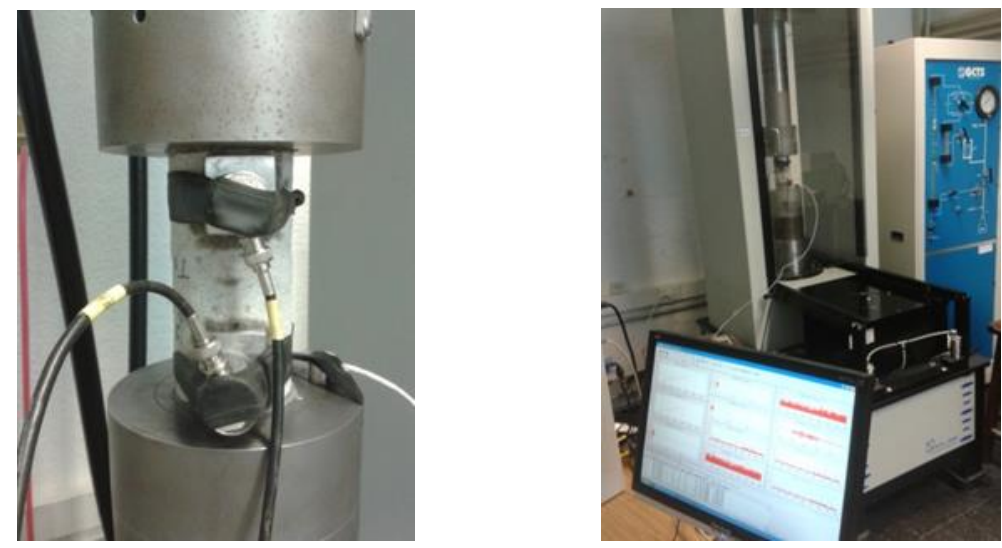

Figura 3: Configuración experimental y equipo de medición de emisión acústica.

Se emplearon tres sensores piezoeléctricos para monitorear la emisión acústica. En la superficie de la probeta se colocaron dos sensores, uno sintonizado en $60 \mathrm{kHz}$ de frecuencia y el segundo en $150 \mathrm{kHz}$. El restante, de banda ancha, se lo adhirió al plato de carga inferior teniendo en cuenta la propiedad de buena guía de onda del metal involucrado. El equipo de emisión acústica se completó con una placa de dos canales PCI-2 de PAC (Physical Acoustic Corporation) y se utilizó el software comercial AEWIN para el análisis y determinación de la mayoría de los parámetros característicos de las señales. Se adoptó como criterio filtrar los eventos de emisión acústica con duración menor a $10 \mu \mathrm{s}$. De esta manera, se redujeron las muestras de varios miles de hits a aproximadamente 50.000 por ensayo, evitando manejar datos irrelevantes de baja representatividad informativa.

En sismología la ley de Gutenberg-Richter (1954) relaciona las señales sísmicas de magnitud A con el número acumulado de eventos sísmicos $\mathrm{N}$ con amplitudes mayores o iguales que A. La dependencia es de tipo potencial y la ley se conoce generalmente en la escala logarítmica-logarítmica como:

$$
N=C \cdot A^{-b} \Rightarrow \log N=a-b \cdot \log A
$$

Esta última es una relación lineal donde a y b son constantes. El exponente b (b-value) representa la proporción relativa de eventos de fractura de diferentes tamaños que progresan durante el daño del material y es la pendiente en la relación lineal.

En nuestro caso, en el análisis de las señales de emisión acústica, $\mathrm{N}$ es el número acumulado de hits de emisión acústica con amplitudes mayores o iguales que A. La toma de datos de la placa del equipo de emisión acústica viene dada por la relación:

$$
A_{d B}=20 \cdot \log \left(A / A_{0}\right)
$$


$\mathrm{A}_{\mathrm{dB}}$ es la amplitud medida en decibeles; A: la amplitud medida en voltios a la salida del sensor piezoeléctrico (sin computar amplificación de la señal) y $\mathrm{A}_{0}=1 \mu \mathrm{V}$ es la unidad de referencia de la amplitud. Finalmente reemplazando la Ecuación (2) en la Ecuación (1) se utilizó en este trabajo la expresión:

$\log N=a-b \cdot\left(A_{d B} / 20\right)$

Es fácil comprobar en la última ecuación que cualquier amplificación de la señal de la amplitud no modifica el valor del b-value.

Se implementó un código con el software MATLAB para determinar a partir de los valores de amplitud de las señales los valores del b-value, estos a su vez se compararon con los obtenidos por el código desarrollado por T. Shiotani e implementado por el software de la Vallen System para la determinación del Ibvalue (improved b-value, mejorado).

\section{RESULTADOS}

El primer resultado obtenido fue corroborar que los datos experimentales se ajustaron a la ley de GutenbergRichter. La Figura 4 muestra el buen ajuste de los datos para la probeta 1- canal 1, se consideraron 33813 eventos filtrados en una duración mayor a $10 \mu$ s resultando un b-value de $b=1.105$ para una duración total del ensayo de aproximadamente 15 minutos y el ajuste fue con un buen coeficiente de correlación de 0.995 . El valor obtenido del b-value es el esperado que oscila entre 0.5 y 1.5. Resultados similares se obtienen para los otros tres ensayos restantes y canales de medición considerados.

El equipo de emisión acústica se preparó, en cada ensayo, para procesar todos los parámetros relevantes de los eventos filtrados. Para cada uno de ellos se obtuvo un gráfico de su comportamiento en función del tiempo para toda la duración de los ensayos. Las Figuras 5 y 6 muestran a modo de ejemplo dos parámetros, el Número de Cuentas y el Rise Time para la probeta 1 y canal 1 . Se indican en el gráfico los puntos que denominaremos característicos donde los parámetros alcanzan algunos valores máximos. Como mostraremos estos tiempos característicos se corresponden con valores mínimos del b-value. En las Figuras 7 y 8 se representan los cambios del b-value en función del tiempo también para la probeta 1 y canal 1 . El criterio adoptado para realizar estos gráficos fue el de calcular el b-value para una misma cantidad de hits por cada punto del gráfico, es decir a tiempos consecutivos se están considerando la misma cantidad de hits. De esta manera, como a medida que progresa el ensayo aumenta la emisión acústica es decir el número de cuentas detectado, los tiempos no están equidistribuidos sino que se acumularán a medida que avance el ensayo y se tendrá un peso adecuado de la emisión acústica por cada punto del gráfico. Como se puede comprobar al comparar tanto la Figura 5 y 6 con la Figura 7 hay coincidencia de algunos valores máximos de los parámetros de emisión acústica con valores mínimos del b-value y esto ocurre en precisamente en lo que denominamos los tiempos característicos. 


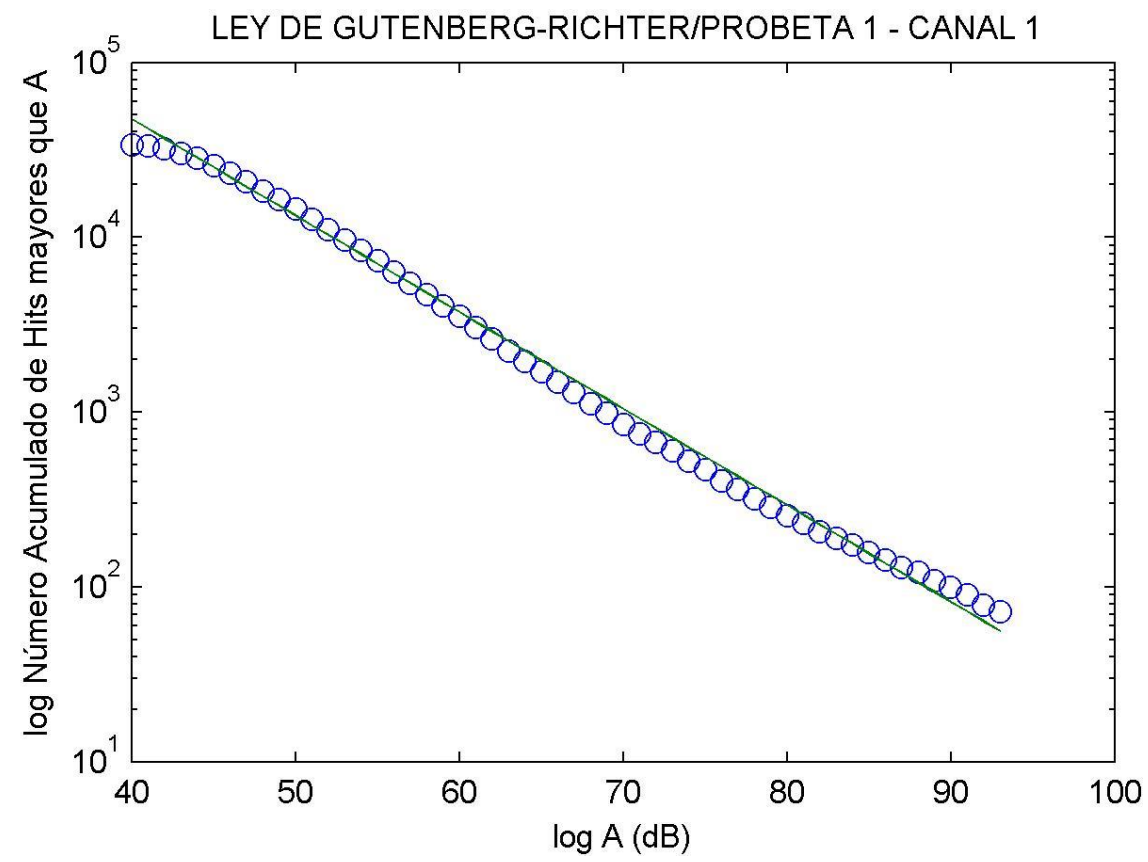

Figura 4: Ley de Gutenberg-Richter que relaciona el número acumulado de eventos de emisión acústica con amplitudes mayores que un dado valor en función de estas amplitudes. La escala del gráfico es del tipo Log-Log. La línea continua es el ajuste lineal por cuadrados mínimos de los datos experimentales para la probeta 1 medidas con el canal 1.

NÚMERO DE CUENTAS vs TIEMPO / PROBETA 1 - CANAL 1

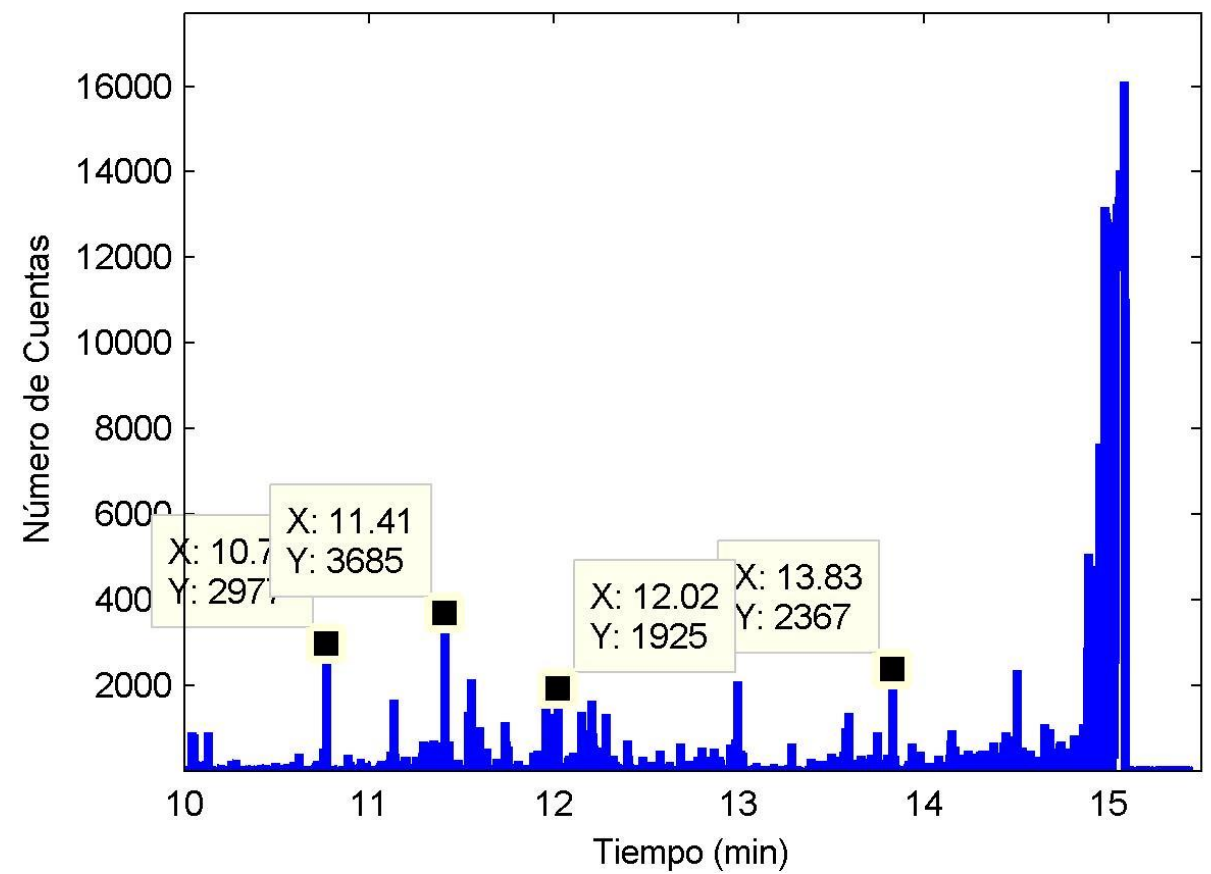

Figura 5: Gráfico del Número de Cuentas de los eventos de emisión acústica en función del tiempo. Se indican los picos característicos que se correlacionan con los valores mínimos del b-value. 


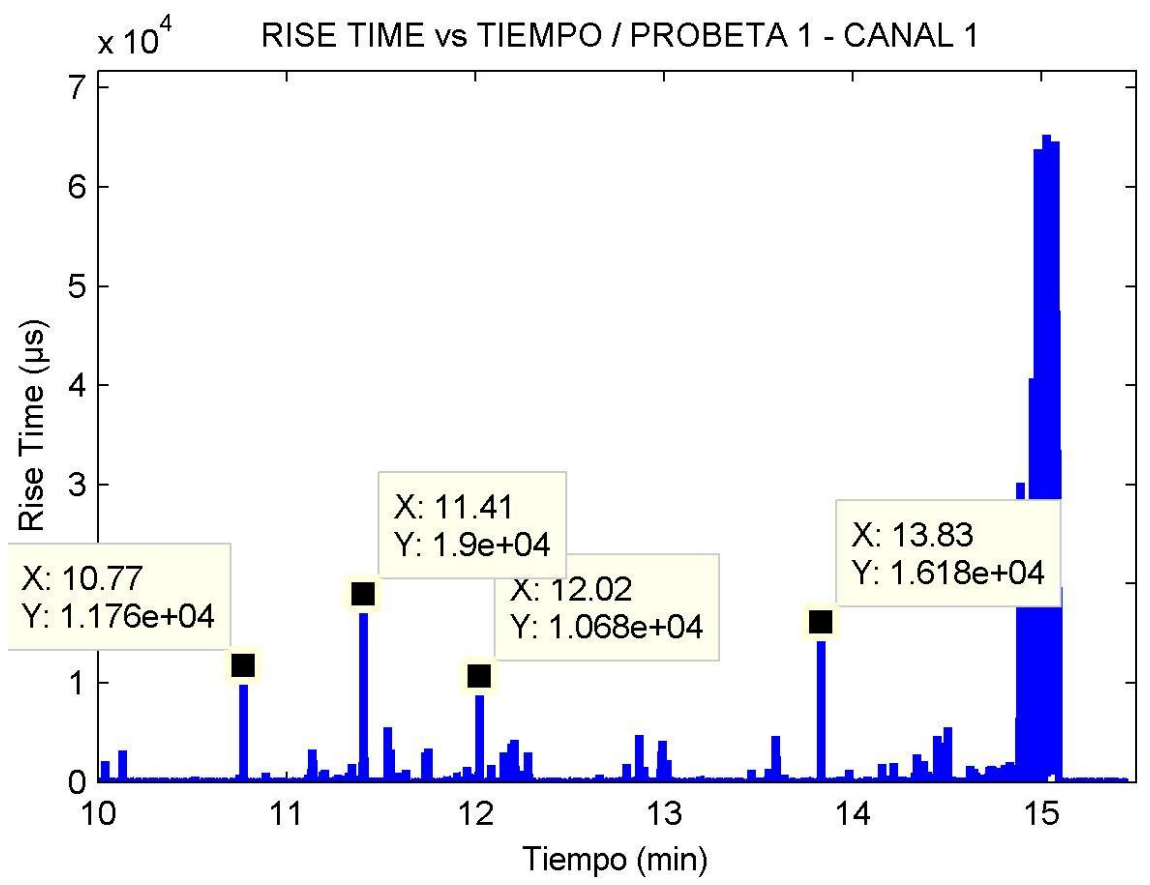

Figura 6: Gráfico del Rise Time (tiempo de subida) de los eventos de emisión acústica en función del tiempo. Se indican los picos característicos que se correlacionan con los valores mínimos del b-value.

b-VALUE EVOLUCIÓN / PROBETA 1 - CANAL 1 / 150 hits por punto

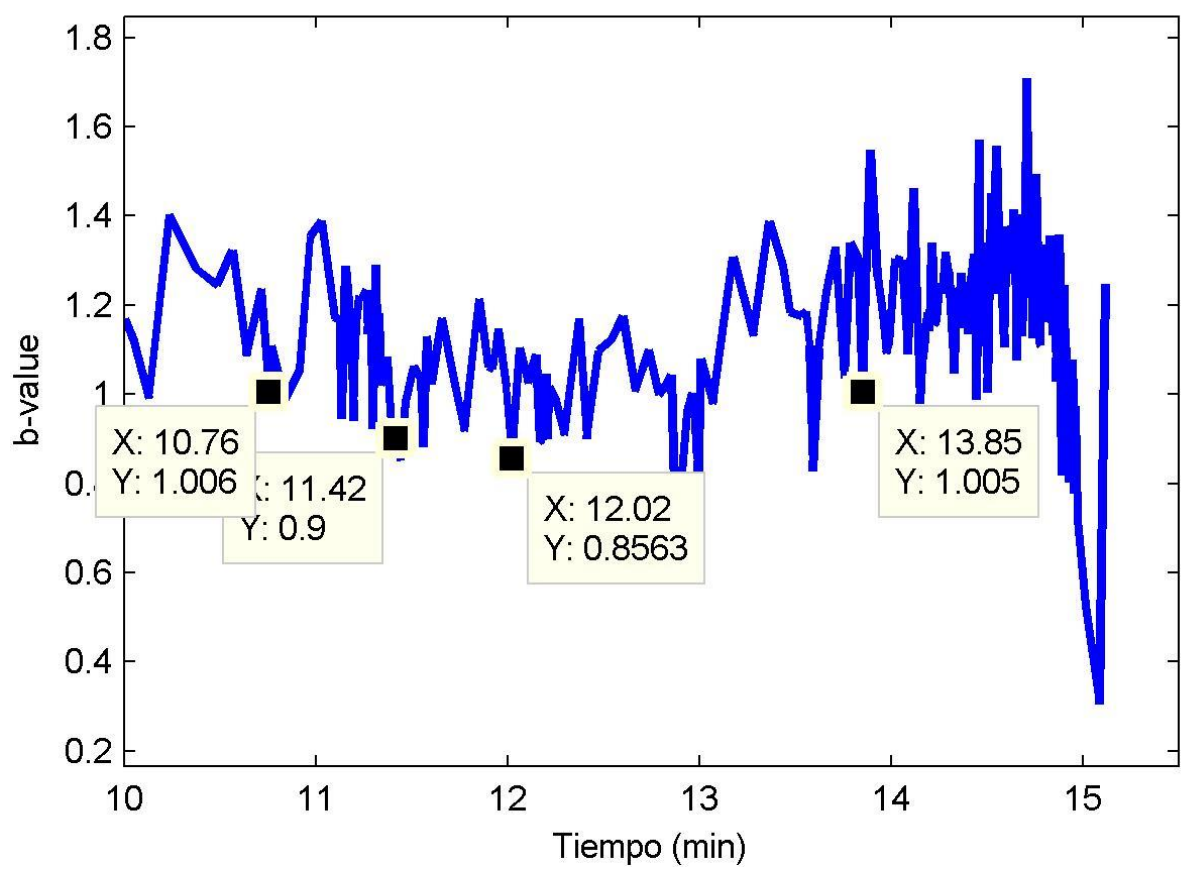

Figura 7: Gráfico de los valores de b-value de los eventos de emisión acústica en función del tiempo para un número relativamente bajo de hits por punto. Se indican los mínimos característicos que se correlacionan con los picos en los valores de los parámetros de emisión acústica. 
b-VALUE EVOLUCIÓN / PROBETA 1 - CANAL 1 / 30 puntos X 1127 hits

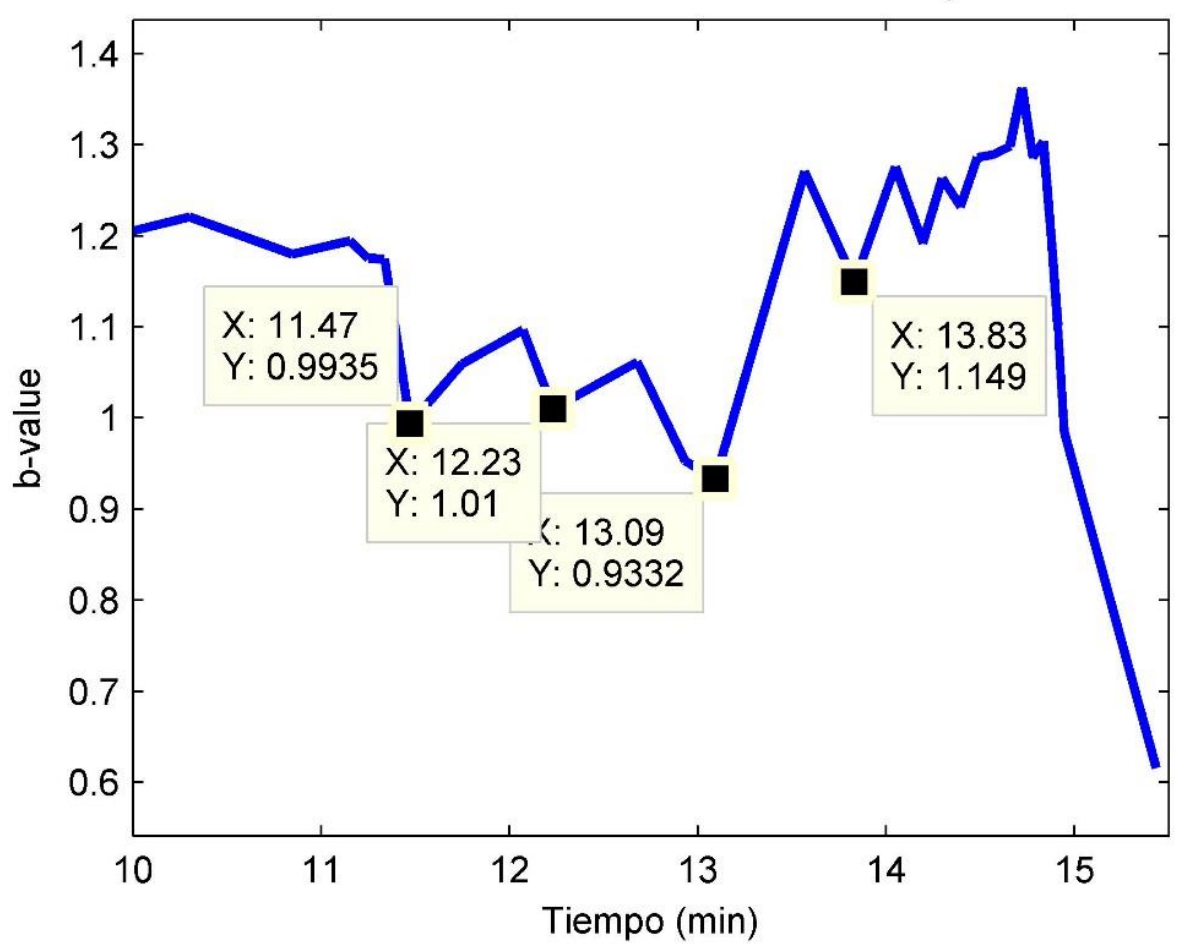

Figura 8: Gráfico de los valores de b-value de los eventos de emisión acústica en función del tiempo para un número relativamente alto de hits por punto. Se indican los mínimos característicos que se correlacionan con los picos en los valores de los parámetros de emisión acústica.

Esta correspondencia se obtiene también para el resto de los parámetros de emisión acústica considerados: energía, valor RMS, duración, etc. Estos tiempos corresponden a porcentajes de la duración total del ensayo de respectivamente: $71 \%, 75,5 \%, 79.5 \%$ y $92 \%$. Es de esperar que estos valores serán característicos para cada material que sea llevado a la rotura y midan el progreso del daño en el mismo, es decir estos valores caracterizan el tipo de rotura de cada material. Podemos asociar estos procesos de daño con las siguientes etapas: a) cierre de grietas pre-existentes y deformación elástica, b) dilatación, c) agrietamiento estable y d) proceso de rotura inestable con coalescencia de micro-grietas y rotura final. Estos resultados obtenidos también se reproducen para cada una de las tres probetas ensayadas y medidas de canal. Podemos asociar estos tiempos característicos con el inicio y fin de cada etapa en el progreso de daño en el material ya que desde el punto de vista de la energía ésta y los parámetros de emisión acústica alcanzan valores máximos que indican cambios en un proceso de daño. Por último se señala que los valores de b-value calculados pueden ser obtenidos de diferente manera al considerar mayor o menor número de puntos en los gráficos, es decir variando la cantidad de hits por puntos. En la Figura 8, comparada con la Figura 7, se calculó el b-value para un número relativamente bajo de puntos, 30 puntos y en consecuencia con un número grande de hits por puntos, esto trae aparejado que en el cálculo se promedia una cantidad grande de eventos y se pierde detalle en el análisis del proceso de daño pero tiene la ventaja que los valores obtenidos presentan menos variaciones o ruido. Se comprobó que estos cambios del cálculo del b-value, en la cantidad de hits por punto, dejan invariantes los tiempos característicos antes señalados, esto debe ser así pues el proceso de daño debe ser independiente del procedimiento de cálculo del b-value. Se obtuvo una mejor correlación entre los máximos de los parámetros y los mínimos del b-value para la probeta 1 y canal 1 cuando se calculó el b-value con una cantidad de 150 hits por punto como se observa en la Figura 7.

\section{CONCLUSIONES}

El análisis de las señales permitió correlacionar el cambio del b-value con los valores máximos relativos de los parámetros de emisión acústica y su comportamiento decreciente, durante un proceso de fisuración, se corresponde con el encontrado en los artículos de trabajos anteriores. Se obtuvo un b-value de $b=1.105$ con un buen coeficiente de correlación de 0.995 . El valor obtenido del b-value es el esperado que oscila entre 0.5 y 1.5. La estimación de los valores de los b-value son adecuados dado que son cualitativamente semejantes al 
obtenido con el software de la Vallen System diseñado por T. Shiotani.

La gran variación de los valores del b-value dificulta el análisis de la señal y se puede modificar buscando un compromiso entre el número de hits considerados en cada tramo (promedio de las variaciones) y el ruido de la señal. Es decir se puede conseguir más detalle de las variaciones pero a costa de aumentar el ruido.

La correlación entre mínimos de b-value y valores máximos de los parámetros de emisión acústica se podría explicar porque en esos puntos indican un aumento de energía del sistema (máximo de los parámetros) seguido por una menor frecuencia acumulada de emisión de eventos dado que el sistema se relaja.

No es posible correlacionar todos los picos que se midieron en los parámetros de emisión acústica con los mínimos en los valores del b-value, así el objetivo de poder, a partir de este estudio, conocer cómo evoluciona el daño en el material durante un proceso de fisuración queda parcialmente cumplido ya que se requeriría de otro estudio para poder reconocer el proceso específico que está ocurriendo en cada etapa identificada.

\section{AGRADECIMIENTOS}

Esta investigación se realiza en el marco de actividades del proyecto ICES (International Centre for Earth Sciences) nodo Argentina, financiado por Comisión de Energía Atómica (CNEA). El autor desea agradecer a la Dra. Ing. Paula Folino del Laboratorio de Métodos Numéricos en Ingeniería (LMNI)-Facultad de Ingeniería-Universidad de Buenos Aires, por su colaboración en las primeras etapas de este trabajo.

\section{BIBLIOGRAFÍA}

[1] GUTENBERG, B., RICHTER, C.F., Seismicity of the Earth and Associated Phenomena, 2nd ed. NJ, USA, Princeton University Press, 1954.

[2] HOEK, E., BIENIAWSKI, Z.T., "Brittle rock fracture propagation in rock under compression", Int. J. Fract. Mech., v. 1, n. 3, pp. 137-155, Sep. 1965.

[3] SCHOLZ, C.H., "Microfracturing and inelastic deformation of rock in compression", J. Geophysical Res., v. 73, n. 4, pp. 1417-1432, Feb. 1968.

[4] LOCKNER, D., "The role of acoustic emission in the study of rock fracture", Int. J. Rock Mech. Min. Sci. \& Geomech., v. 30, n. 7, pp. 883-889, 1993.

[5] SHIOTANI, T., FUJI, K., AOKI, T., AMOU, K., "Evaluation of progressive failure using AE sources and improved b-value on slope model test", Prog. Acoust. Emission, 7, pp. 529-534, 1994.

[6] RAO, M.V.M.S., "Significance of AE based b-value in the study of progressive failure of brittle rock: some examples from recent experiments", In: Proceedings of the 14th World Conference on Non-Destructive Testing (14th WCNDT), 4, pp. 2463-2467, New Delhi, India, 1996.

[7] SHIOTANI, T., YUYAMA, S., LI, Z. W, OHTSU, M., "Application of the AE improved b-value to qualitative evaluation of fracture process in concrete materials" J. Acoust. Emission, 19, pp. 118-132, 2001.

[8] RAO, M.V.M.S., PRASANNA LASKHMI, K. J., "Analysis of b-value and improved b-value of acoustic emissions accompanying rock fracture”, Current Science, v. 89, n. 9, pp. 1577-1582, Nov. 2005.

[9] CARPINTERI, A., LACIDOGNA, PUZZI, G.S., "From criticality to final collapse: Evolution of the "bvalue" from 1.5 to 1.0", Chaos, Solitons and Fractals, 41, pp. 843-853, 2009.

[10] FILIPUSSI, D.A., GUZMÁN, C., XARGAY, H., TORRES D.N., "Study of Acoustic Emission in a Compression Test Andesite Rock", Procedia Materials Science, 9, pp. 292-297, 2015. 